\title{
Analysis of Polycerate Mutants Reveals the Evolutionary Co-option of HOXD1 to Determine the Number and Topology of Horns in Bovidae
}

Aurélie Allais-Bonnet ${ }^{1,2,3 \#}$, Aurélie Hintermann",\#, Marie-Christine Deloche ${ }^{1,2,3}$, Raphaël Cornette $^{5}$, Philippe Bardou ${ }^{6,7}$, Marina Naval-Sanchez ${ }^{8}$, Alain Pinton ${ }^{6}$, Ashleigh Haruda ${ }^{9}$, Cécile Grohs $^{10}$, Jozsef Zakany ${ }^{4}$, Daniele Bigi ${ }^{11}$, Ivica Medugorac ${ }^{12}$, Olivier Putelat ${ }^{13,14}$, Ockert Greyvenstein $^{15}$, Tracy Hadfield ${ }^{16}$, Slim Ben Jemaa ${ }^{17}$, Gjoko Bunevski ${ }^{18}$, Fiona Menzi ${ }^{19}$, Nathalie Hirter ${ }^{19}$, Julia M. Paris ${ }^{19}$, John Hedges ${ }^{20}$, Isabelle Palhiere ${ }^{6}$, Rachel Rupp ${ }^{6}$, Johannes A. Lenstra ${ }^{21}$, Louisa Gidney ${ }^{22}$, Joséphine Lesur ${ }^{23}$, Renate Schafberg ${ }^{9}$, Michael Stache ${ }^{9}$, MarieDominique Wandhammer ${ }^{24}$, Rose-Marie Arbogast ${ }^{25}$, Claude Guintard ${ }^{26,27}$, Amandine Blin ${ }^{28}$, Abdelhak Boukadiri ${ }^{10}$, Julie Riviere ${ }^{10,29}$, Diane Esquerré $^{30}$, Cécile Donnadieu ${ }^{30}$, Coralie Danchin-Burge ${ }^{31}$, Coralie M Reich ${ }^{32}$, David Riley ${ }^{15}$, Este van Marle-Koster ${ }^{33}$, Noelle Cockett ${ }^{16}$, Benjamin J. Hayes ${ }^{34}$, Cord Drögemüller ${ }^{19}$, James Kijas ${ }^{8}$, Eric Pailhoux ${ }^{2,3}$, Gwenola TosserKlopp $^{6}$, Denis Duboule ${ }^{4,35,36, *}$ and Aurélien Capitan ${ }^{1,10, *}$

${ }^{1}$ ALLICE, 75012 Paris, France

${ }^{2}$ Université Paris-Saclay, UVSQ, INRAE, BREED, 78350, Jouy-en-Josas, France

${ }^{3}$ Ecole Nationale Vétérinaire d'Alfort, BREED, 94700, Maisons-Alfort, France

${ }^{4}$ Department of Genetics and Evolution, University of Geneva, 1211, Geneva 4, Switzerland

${ }^{5}$ Institut de Systématique, Evolution, Biodiversité (ISYEB), Muséum National d'Histoire Naturelle, CNRS, Sorbonne Université, EPHE, Université des Antilles, CP50, 75005 Paris, France

${ }^{6}$ GenPhySE, Université de Toulouse, INRAE, ENVT, F-31326, Castanet Tolosan, France

${ }^{7}$ INRAE, Sigenae, Castanet-Tolosan, France

${ }^{8}$ CSIRO Agriculture \& Food, St. Lucia, 4067, QLD, Australia

${ }^{9}$ Central Natural Science Collections, Martin Luther University Halle-Wittenberg, 06108 Halle (Saale), Germany

${ }^{10}$ Université Paris-Saclay, INRAE, AgroParisTech, GABI, 78350 Jouy-en-Josas, France

${ }^{11}$ Dipartimento di Scienza e Tecnologie Agro-Alimentari, Alma Mater Studiorum University of Bologna, Bologna, Italy

${ }^{12}$ Population Genomics Group, Department of Veterinary Sciences, Ludwig-MaximiliansUniversity Munich, Munich, Germany

${ }^{13}$ Archéologie Alsace, 67600 Sélestat, France 
bioRxiv preprint doi: https://doi.org/10.1101/2020.11.04.363069; this version posted November 4,2020 . The copyright holder for this preprint (which was not certified by peer review) is the author/funder, who has granted bioRxiv a license to display the preprint in perpetuity. It is made available under aCC-BY-NC-ND 4.0 International license.

${ }^{14}$ UMR 7044 - ARCHIMEDE - Misha, 67083 Strasbourg, France

${ }^{15}$ Department of Animal Science, Texas A\&M University, College Station, TX, 77843, USA

${ }^{16}$ Department of Animal, Dairy, and Veterinary Sciences, Utah State University, Logan, UT, 84322-4700, USA

${ }^{17}$ Laboratoire des Productions Animales et Fourragères, Institut National de la Recherche Agronomique de Tunisie, Université de Carthage, Ariana, Tunisia

${ }^{18}$ Livestock Department, Faculty of Agricultural Sciences and Food Institute of Animal Biotechnology, University Ss. Cyril and Methodius, 1000 Skopje, North Macedonia

${ }^{19}$ Institute of Genetics, Vetsuisse Faculty, University of Bern, Bern, 3001, Switzerland

${ }^{20}$ Manx Loaghtan Sheep Breeders' Group, Spring Lane, Bassingbourn, Cambridgeshire, SG8 5HT, UK

${ }^{21}$ Utrecht University, Faculty of Veterinary Medicine, Utrecht, Netherlands

${ }^{22}$ Rent a Peasant, Tow Law, Bishop Auckland, Durham County, DL13 4BB, UK

${ }^{23}$ Unité Archéozoologie, Archéobotanique, Sociétés Pratiques et Environnements (AASPE), CNRS, Muséum National d'Histoire Naturelle, 75005 Paris, France

${ }^{24}$ Musée Zoologique de Strasbourg, 67000 Strasbourg, France

${ }^{25}$ CNRS UMR 7044 - ARCHIMEDE - Misha, 67083 Strasbourg, France

${ }^{26}$ Unité d'Anatomie Comparée, Ecole Nationale Vétérinaire de l'Agroalimentaire et de l'Alimentation, Nantes Atlantique - ONIRIS, 44307 Nantes, France

${ }^{27}$ Groupe d'Études Remodelage osseux et bioMatériaux (GEROM), Université d'Angers, Unité INSERM 922, LHEA/IRIS-IBS, CHU d'Angers, 49100 Angers, France

${ }^{28}$ Muséum National d'Histoire Naturelle, CNRS, UMS 2700 2AD, CP 51,75005 Paris, France

${ }^{29}$ INRAE, Micalis Institute, AgroParisTech, Université Paris-Saclay, 78350 Jouy-en-Josas, France

${ }^{30}$ INRAE, US 1426, GeT-PlaGe, Genotoul, Castanet-Tolosan, France

${ }^{31}$ Institut de l'Elevage, 75012 Paris, France

${ }^{32}$ Agriculture Victoria, AgriBio, Centre for AgriBioscience, Bundoora, Victoria, Australia

${ }^{33}$ Department of Science, University of Pretoria, P/bag X20 Hatfield, 0028, South Africa

${ }^{34}$ Queensland Alliance for Agriculture and Food Innovation (QAAFI), Centre for Animal Science, University of Queensland, St. Lucia, QLD, 4072, Australia

${ }^{35}$ Swiss Cancer Research Institute, EPFL, Lausanne, Switzerland

${ }^{36}$ Collège de France, Paris, France

\# These authors contributed equally to this work 
* Corresponding authors:

69 Aurélien Capitan

70 Université Paris-Saclay, INRAE, AgroParisTech, GABI, 78350 Jouy-en-Josas, France

71 aurelien.capitan@inrae.fr

72

73 Denis Duboule

74 Department of Genetics and Evolution, University of Geneva, 1211, Geneva 4, Switzerland

75 Swiss Cancer Research Institute, EPFL, Lausanne, Suisse

76 Collège de France, Paris, France

77 denis.duboule@epfl.ch

78

79 Key words

80 Hox genes, co-option, regulatory mutation, goat and sheep genomics 


\section{Abstract}

In the course of evolution, pecorans (i.e. higher ruminants) developed a remarkable diversity of osseous cranial appendages, collectively referred to as 'headgear', which likely share the same origin and genetic basis. However, the nature and function of the genetic determinants underlying their number and position remain elusive. Jacob and other rare populations of sheep and goats, are characterized by polyceraty, the presence of more than two horns. Here, we characterize distinct POLYCERATE alleles in each species, both associated with defective HOXD1 function. We show that haploinsufficiency at this locus results in the splitting of horn bud primordia, likely following the abnormal extension of an initial morphogenetic field. These results highlight the key role played by this gene in headgear patterning and illustrate the evolutionary co-option of a gene involved in the early development of bilateria to properly fix the position and number of these distinctive organs of Bovidae.

\section{Introduction}

In pecorans, successive environmental and behavioural adaptations favoured the emergence and sometimes the secondary loss of a variety of headgear, as exemplified by bovid horns, cervid antlers, giraffid ossicones or antilocaprid pronghorns (Davis et al. 2011; Wang et al. 2019). As different as they are, these iconic organs share both a common cellular origin and a minimal structural organisation: they derive from neural crest stem cells and consist of paired structures, located on the frontal bones and composed of a bony core covered by integument (Davis et al. 2011; Wang et al. 2019) (Fig. 1, Suppl. Fig. 1). While the development and evolution of headgear is a long-standing question, the underlying molecular and cellular mechanisms have been difficult to study, mostly because the patterning and differentiation of headgear progenitor cells occur early during embryogenesis (Lincoln 1973; Allais-Bonnet et al. 2013) and involve hundreds of genes (Wang et al. 2019).

In this context, natural mutations affecting headgear number, shape or position, such as the polycerate (multi-horned) phenotype occurring in small ruminants (Fig. 1a, b, OMIA 0008069940), offer a valuable alternative (Capitan et al. 2012). Polyceraty was already observed in the oldest ovine remains from Çatalhöyük, Turkey (ca 6000 BCE(Epstein 1971; Putelat 2005)) and this dominant phenotype currently segregates in several sheep breeds around the world. Even though the corresponding locus was mapped in seven distinct populations to the same region of chromosome 2 (Chr2), it has not yet been identified (Greyvenstein et al. 2016; He et al. 2016; Kijas et al. 2016; Ren et al. 2016). In contrast, polycerate goats are found only sporadically in 
the Alps and have not been subject to any genetic studies thus far. The oldest record dates back from 1786, when the French Queen Marie-Antoinette imported a four-horned billy-goat from the city of Bulle, Switzerland to her model farm (Heitzmann 2006).

We set up to determine the genetic bases of these conditions in sheep and goats and, in this study, we show that polyceraty in Bovidae is due either to a four-base-pair deletion affecting the splicing of the Hoxdl gene in sheep, or to the deletion of a large regulatory region controling the same gene, in goats. These results thus illustrate the evolutionary co-option of this gene normally involved in early development to help determine the position and number of horns. They also show that comparable phenotypes observed in distinct species and selected and maintained for a long time are caused by the mis-regulation of the same gene.

\section{Results and Discussion}

\section{Characterisation of POLYCERATE Mutations in Sheep and Goats}

To identify the molecular determinants of polyceraty, we re-analysed the Illumina OvineHD Beadchip genotyping data (600 k SNPs) of 111 case and 87 control sheeps generated by two previous studies (Greyvenstein et al. 2016; Kijas et al. 2016) (Suppl. Tables 1 and 2). Assuming autosomal dominant inheritance and genetic homogeneity in the three breeds investigated, we fine-mapped the ovine POLYCERATE locus between positions 132,717,593 and 133,151,166 -bp on Chr2 (Suppl. Fig. 2). By comparing whole genome sequences of 11 polycerate specimens and 1'179 controls representing the world-wide sheep diversity, we identified a single candidate variant in this interval: a four-nucleotide deletion located at position +4 to +7 bp after exon 1 of the $H O X D 1$ gene (g.132,832,249_132,832,252del; Fig. 1c). Genotyping of this variant in 236 animals from eight populations containing polycerate specimens showed a perfect genotype to phenotype association (Suppl. Tables 3 and 4). Moreover, cross-species alignments revealed that the +4 and +5 nucleotides are conserved amongst 103 sarcopterigian and tetrapod species, supporting an important role in the splicing of HOXD1 precursor RNAs (Fig. 1c and Suppl. Table 5).

We next mapped the caprine POLYCERATE locus to a $542 \mathrm{~kb}$ large region orthologous to that of the ovine locus (Chr2:115,143,037-115,685,115 bp on ARS1 assembly (Bickhart et al. 2017); Suppl. Fig. 2), by using a panel of 35 polycerate and 51 two-horned goats obtained from eight European populations and genotyped with the Illumina GoatSNP50 BeadChip (TosserKlopp et al. 2014) (Suppl. Table 6). Within this interval, we identified 36 private heterozygous variants in one heterozygous polycerate goat versus 1'160 control individuals (Suppl. Table 
7). Genotyping of five case-control pairs from distinct breeds reduced the list of candidates to 15 short variants, affecting genomic regions not conserved amongst 103 eutherian mammals, as well as a rare type of structural variation located $57 \mathrm{~kb}$ downstream of the HOXD1 3'UTR (Suppl. Tables 7 and 8). The latter involved the translocation of $137 \mathrm{~kb}$ from $\mathrm{Chr} 5$ to $\mathrm{Chr} 2$ by means of a circular intermediate (Durkin et al. 2012) and the deletion of $503 \mathrm{~kb}$ from the insertion site (g.115,652,290_116,155,699delins137kb; Fig. 1d, e and Suppl. Fig. 3). Consequently, the mutant chromosome lacked the MTX2 gene and carried an exogenic copy of both $R A S S F 3$ and the first ten exons of GNS. Genotyping of this variant in 77 case and 355 control goats originating from 24 distinct populations revealed a 100 percent association between polyceraty and heterozygosity for the large insertion/deletion (Suppl. Table 9 and

Suppl. Fig. 4). Homozygous mutants were not detected in our panel, whereas at least 14 polycerate animals were born from polycerate pairs of parents (binomial $\mathrm{p}=3.4 \times 10^{-3}$; Suppl. Note 1). Because the knockdown of Mtx2 in zebrafish is embryonic lethal at gastrulation (Wilkins et al. 2008) and newborn mice homozygous for a deletion including Mtx2 were never scored (binomial $\mathrm{p}=5.7 \times 10^{-6}$; Suppl. Note 1), we concluded that homozygosity at the goat POLYCERATE locus is an early lethal condition.

\section{Remote Hoxd1 regulation in Transgenic Mice}

These mapping studies identified the HOXD gene cluster as being involved in the polycerate phenotype in both sheep and goats. This cluster contains nine homeobox genes encoding transcription factors involved in the organisation of the body plan during embryogenesis (Krumlauf 1994). Both their timing of activation and their domains of expression are determined by their respective positions along the gene cluster (Kmita and Duboule 2003). Accordingly, the mouse Hoxdl gene is expressed very early on and in the most rostral part of the embryo (Fig. 2a). In rodents, Hoxdl is expressed in crest cell-derived head structures (Frohman and Martin 1992), which made this gene a particularly interesting candidate for polyceraty. Also, a DNA sequence conserved only amongst pecorans species carrying headgear was identified $15 \mathrm{~kb}$ downstream of HOXDl ("HCE" in (Wang et al. 2019)). This sequence, however, is not included in the large insertion-deletion observed in polycerate goats.

We assessed whether the deletion present in goat may impact the expression of HOXD1 in cranial crest cells by looking at a series of modified mouse strains either carrying transgenes or where a targeted deletion was induced at the orthologous locus (see Methods). First, the wide presence of cells expressing Hoxdl both in the face and in the cranial derma, the latter being of crest cell origin, was detected in fetuses with a targeted integration of lacZ sequences into the 
Hoxd1 gene (Fig. 2b, Hoxd1 $1^{\text {Lac }}$ ). Expression was however not scored in the crown region (Fig. $\mathbf{2 b}$, dashed circle), the area corresponding to that of horn bud differentiation in Bovidae (Dove 1935; Capitan et al. 2011). Instead, Hoxd1 was expressed abundantly in other regions of the head including the eyelids (Fig. 2b, white arrow and arrowhead), an observation consistent with the abnormal upper eyelids and eyebrows often detected in ovine and caprine polycerate animals (Suppl. Fig. 5-7).

We next tried to localize the underlying regulatory elements by using transgenic BACs with lacZ sequences introduced within Hoxd1. A BAC covering the HoxD cluster itself did not show any expression in the head, suggesting that regulatory sequences are not located in the gene cluster (Fig. 2b, $\operatorname{Tg} B A C^{H o x D}$ ). In contrast, a transgenic BAC extending in the region upstream of Hoxd1 and including Mtx2 gave a staining similar to $\operatorname{Hoxd1}^{\operatorname{Lac}}$ (Fig. 2b, $\operatorname{Tg} B A C^{M t x 2}$ ), indicating that regulatory sequences were located upstream Hoxd1, in a region including and surrounding Mtx2. The latter result was controlled by using an engineered $151 \mathrm{~Kb}$ deletion of a largely overlapping region, including a lacZ reporter gene, which expectedly abrogated Hoxd1 expression in cranial cellular populations (Fig. 2b, HoxD $D^{\text {Del( }(151 k b) l a c}$ ). As a positive control for the lacZ reporter system, expression of Hoxdl in neural derivatives driven by sequences within the HoxD cluster was scored, as expected (Fig. 2b, black arrows). These analyses in mice demonstrated that regulatory sequences driving Hoxdl expression in the head are located in a region largely comprised within the deletion determined in goats as causative of polyceraty, further suggesting that the latter deletion abrogates $H O X D 1$ expression in goat fetuses.

\section{Expression of HOXD1 in Pecoran Fetuses}

To investigate whether the abence of mouse Hoxdl expression in the crown region of the head was also observed in Pecoran embryos, we isolated heterozygous polycerate and wild type fetuses both at $70 \mathrm{dpc}$ (days post-coïtum) in goat and at $76 \mathrm{dpc}$ in sheep, two stages where eyelids are fully grown and horn buds can be distinguished (Suppl. Fig. 8). After microdissection and Reverse Transcription quantitative PCR (RT-qPCR), we noticed that in wild type fetuses of both species, HOXD1 expression was significantly lower in horn buds than in surrounding tissues (Fig. 3a, b), reminiscent of the weak -if any- expression of Hoxdl observed in a comparable region in the mouse. In heterozygous mutant goat fetuses, however, HOXD1 RNA levels were equally low in all three samples (Fig. 3a), re-enforcing the idea that the caprine POLYCERATE variant negatively affects the expression of HOXD1. 
In sheep, despite some variation due to slight differences in sampling (Fig. 3b, upper histogram and methods), heterozygous mutants for the four-base-pair deletion adjacent to the splice donor site displayed RNA amounts roughly similar to control specimens when primers targeting the second exon of the gene were used. However, RT-qPCR with intronic primers revealed significant intron retention in all mutant tissues but horn buds, where expression was likely too low (Fig. 3b lower histogram). Intron retention is predicted to result in a non-functional protein, truncated two residues after the last amino acid encoded by exon 1 and thus lacking the DNA binding peptide (Suppl. Fig. 9). Therefore, both POLYCERATE variants appear to reduce the amount of functional HOXD1 RNAs in the horn bud region, likely leading to a loss of boundary condition and an extension of the cellular field permissive for horn bud development. This extension would elongate the bud region sufficiently to split it into two separate organs.

\section{Morphometric Analyses and Topology of the Horn Field}

To substantiate this hypothesis, we analysed variations in horn topology in 61 ovine and 19 caprine skulls from various populations using 3D geometric morphometrics (Suppl. Table 10). We performed Principal Component Analysis (PCA) using 116 landmarks and sliding semilandmarks after removing non-shape variation (see Methods). We then plotted the first principal components (PCs) to visualize the specimen distribution in the morphospace (Fig. 4a, Suppl. Fig. 10-12 and Suppl. Table 11). The first two axes represented 35.8\% and 23.3\% of the total variance and distinguished the phenotype and species categories, respectively. Along the first axis, we individualized three sub groups of polycerate specimens in sheep, based on the distances between lateral horns (dlh, Fig. 4a). Of note, the group displaying the largest dlh (i.e. that with the most negative values along the $\mathrm{x}$ axis) had no equivalent in goat, possibly due to early lethal homozygosity (see above).

We looked at the association between genotypes and horn implantation within polycerate animals by measuring the distances both between the lateral horns on the left side of the skull (dlhl) and between the upper horns (duh) in 29 rams (Suppl. Table 12). We found a significant difference in the proportions of homozygous and heterozygous specimens in animals with dlhl $\leq$ duh versus dlhl $>$ duh (Fig. 4 b) and no heterozygous animal was found to have dlhl $>$ duh. We computed the theoretical skull shape at the maximum and minimum of PC1 axis (Fig. 4c) and the corresponding vectors of deformation (Fig. 4d). The results obtained were consistent with a splitting of horn buds in polycerate animals. This splitting always occurred along the major axis of the ellipse formed by the wild type horn bud, with an extension of the hemi-horn buds in an area where HOXD1 expression was detected in wild type specimens (Fig. 4d and 
above). In homozygous animals, the new cellular field was likely larger than in heterozygous, leading to a clearer separation of hemi-horns, whereas heterozygous specimens often displayed partially fused organs. This is markedly different from the production of additional horns, as observed in subspecies of Tetracerus quadricornis (Groves 2003) (Suppl. Fig. 13).

\section{Conclusions}

From these results, we conclude that pecorans have an intrinsic capacity to induce hornbuds within a presumptive head territory. This capacity appears to be associated with the nonexpression of the HOXDI transcription factor, which is present in surrounding cells and may delimit this field, a function somewhat distinct from the ancestral role of Hox genes during development (Krumlauf 1994). Various haploinsufficient conditions lead to the extension of this territory, a condition fully achieved in complete absence of HOXD1. While a weak extension of this morphogenetic field leads to the growth of twin horns, fused at their bases, a full extension induces the complete splitting of the horn bud, thus generating a pair of lateral horns. We hypothesize that the initial expression of HOXD1 in anterior crest cells made this evolutionary co-option possible and thus helped to determine the position and number of horns, which became the distinctive trait of Bovidae.

\section{Materials and Methods}

\section{Ethics Statement}

All experiments reported in this work comply with the ethical guidelines of both the French National Research Institute for Agriculture, Food and Environment (INRAE) and the University of Geneva, Switzerland. Blood samples were collected on sheep and goats during routine blood sampling (for annual prophylaxis, paternity testing or genomic selection purpose) by trained veterinarians and following standard procedures and relevant national guidelines. Sample collection of small ruminants in Switzerland was approved by the Cantonal Committee for Animal Experiments (Canton of Bern; permit 75/16). Ovine and caprine fetuses were produced in an INRAE experimental farm (Bressonvilliers, France) and collected in the INRAE experimental slaughterhouse of Jouy-en-Josas (France). Experiments were performed in strict accordance with the European directive 2010/63/UE and were approved by the local Institutional Animal Care and Use Committee of AgroParisTech/INRAE (COMETHEA, permit number 19/032). All experiments with mice were performed in agreement with the Swiss law on animal protection (LPA), under license No GE 81/14 (to D.D.). All the samples and data 
analyzed in the present study were obtained with the permission of breeders, breeding organizations and research group providers.

\section{Animals}

Live sheep and goats. Sheep and goats from a wide diversity of breeds around the world were involved in at least one of the analyses performed in this study. Briefly, they felt into four categories: 1) animals genotyped with Illumina OvineHD or GoatSNP50 (Tosser-Klopp et al. 2014) BeadChip for mapping the POLYCERATE locus in both species (Supplementary Tables 1 and 6); 2) a set of whole genome sequences used for identifying and filtering candidate mutations (Supplementary Table 13); 3) animals genotyped by PCR and Sanger sequencing for candidate mutations (Supplementary Tables 3 and 7); and 4) polycerate sheep animals genotyped for verifying putative differences between heterozygous and homozygous individuals in terms of distances between the lateral horns and between the upper horns (Supplementary Table 12).

Mouse models. Five different transgenic mouse stocks were used (see Supplementary Table 14). The HoxD $D^{(D e l 365)}$ allele was produced by CRISPR-Cas 9 technology. sgRNA were designed manually, ordered as DNA oligos at Eurogentec and cloned into px330. sgRNAs were synthetized with HiScribe T7 high yield RNA synthesis kit (New England Biolabs), incubated together with Cas9 mRNA and then electroporated into fertilized mouse zygotes (see also Supplementary Note 1). The HoxD ${ }^{(\text {Dell5I) }}$ allele was obtained by using CRE-mediated recombination (Andrey et al. 2013). The Transgenic fetuses from four strains containing different lacZ constructions were collected from stage E12.5 to E.15.5. The Hoxd $1^{L a c}$ strain was obtained by inserting a LacZ cassette in the HindIII site of the second exon of Hoxdl (Zakany et al. 2001). The $\mathrm{BAC}^{H o x D}$ and $\mathrm{BAC}^{M t x z}$ result from the introduction of a LacZ-SV40promoterHoxd1-zeocin cassette in the HindIII site of the second exon of Hoxdl (Schep et al. 2016). These BACs were selected based on their localization on the physical map of the mouse genome (Gregory et al. 2002) and obtained from the RPCI-23 and -24 Mouse (C57BL/6J) BAC Libraries from the Children's Hospital Oakland Research Institute (https://bacpacresources.org/libraries.php). The modified BACs were purified, linearised and microinjected into mouse fertilised oocytes to obtain each of these strains in mixed Bl6XCBA hybrid background, by standard procedures. Gene expression analyses were performed on heterozygous specimens. 
A precise map of the orthologous Hoxd region in mouse and goat was obtained by aligning on murine GRCM38/mm10 genome assembly the BAC end sequences and goat genome sequences of $10 \mathrm{~kb}$ segments encompassing the breakpoints of the large insertion-deletion. Alignments were carried out using the BLAT tool from the UCSC Genome Browser (http://genome.ucsc.edu/cgi-bin/hgBlat).

Animals subject to post-mortem clinical examination. The eyelids and eyes fundus were examined in a 3-weeks old polycerate male Provençale kid who died from a natural cause and a matched control, as well as in an 8-year old polycerate Jacob ewe and her wild type half-sister after slaughter.

Ovine and Caprine fetuses were generated by mating heterozygous polycerate males of the caprine Provençale and ovine Jacob breeds with wild type cull females, after oestrus synchronization. Oestrus cycles were synchronized using intravaginal sponge impregnated with progestagen for 15 days followed by PMSG (Pregnant Mare Serum Gonadotropin) injection 48 $\mathrm{h}$ after sponge removal. Pregnant females were anaesthetized by electronarcosis and euthanized by immediate exsanguination on day 70 or 76 post-coïtum in the INRAE slaughterhouse of Jouy-en-Josas (France). Directly after, the fetuses were recovered from their genital tracts and exsanguinated. 'Skin' samples comprising the epidermis, dermis and hypodermis were collected at different locations on the left side of the head of the $70 \mathrm{dpc}$ goat and $76 \mathrm{dpc}$ sheep fetuses (see Fig. 3) for expression studies. Of note, the skin of the back of the head was sampled slightly more caudally in polycerate animals due to the specific localization of the posterior pair of horns. The same skin samples were collected on the right side of the head with the underlying bone for histological analyses. Four case fetuses and four controls of matched sex were selected in each species for expression studies. Finally, for verification, liver samples were also collected for DNA extraction and subsequent genotyping of the fetuses for the sheep and goat POLYCERATE mutations.

Skull specimens. The skulls from 61 sheep (32 polycerate, 29 wild type) and 19 goats (12 polycerate, 7 wild type) were obtained from different anatomical collections. These specimens were sampled over the last 170 years and originate from a wide variety of populations. Information on horn phenotype, species, gender, age, population or breed, collection, and year of entry in the collection are presented in Supplementary Table 10.

\section{Phenotyping}


The polycerate phenotype is an autosomal dominant trait readily visible on fetuses at $70 \mathrm{dpc}$ in goat and $76 \mathrm{dpc}$ in sheep (Supplementary Fig. 8). Phenotyping at birth is difficult due to the presence of hairs and it is necessary to wait for after the first month to distinguish horns growing amid fur. In polycerate animals, horns have a nearly circular cross section but, depending on their relative placement, they may progressively fuse at the base with other horns located on the same side of the skull. The growth in width of horns is expected to affect the measure of distances between the lateral horns (dlh) and the upper horns (duh) but not their relative sizes. This, together with the fact that we never observed any case of fusion between the upper horns led us to consider the dlh/duh ratio on the left side of the head to distinguish different types of four-horned animals in one of the analyses performed in this study. Polyceraty is frequently associated with defects of the eyelid in both species. While we did not systematically record this particular phenotype, we performed post-mortem clinical examination of the eyelids and eyebrows in one case and one control animal per species (Supplementary Fig. 5-7).

\section{DNA Extraction}

Ovine and caprine DNAs were extracted from hair root, blood or liver samples using the DNeasy Blood and Tissue Kit (Qiagen). Murine DNA was isolated from ear snip after Proteinase K digestion using standard phenol/chloroform protocol. DNA quality was controlled by electrophoresis and quantified using a Nanodrop spectrophotometer (Thermo Scientific).

\section{IBD-Mapping of Caprine and Ovine POL YCERATE Loci}

General principle. Assuming autosomal dominant inheritance and genetic homogeneity in each of the species investigated, all polycerate animals share at least one copy of the same causative mutation and of a surrounding chromosomal segment inherited-by-descent from a common ancestor. Therefore, comparing SNP array genotyping data of two distantly related polycerate animals is expected to reveal a number of Mendelian incompatibilities (i.e. homozygosity for different alleles) throughout their genomes but not within shared IBD segments. Accordingly, we screened Mendelian incompatibilities in all the possible pair combinations of polycerate $\mathrm{x}$ polycerate $(4 \mathrm{H} 4 \mathrm{H}$ pairs) and polycerate $\mathrm{x}$ wild type $(4 \mathrm{H} 2 \mathrm{H})$ individuals. Pairs with a proportion of Medelian incompatibilities below 1 percent of the total number of markers tested were declared as constituted of parent and offspring and were not considered in the analysis. Then, for sliding windows of $\mathrm{n}$ markers ( $\mathrm{n}$ set to 10 in goat and 50 in sheep considering differences in marker density) we scored the numbers of $4 \mathrm{H} 4 \mathrm{H}$ pairs and $4 \mathrm{H} 2 \mathrm{H}$ pairs for which 'no' versus 
'at least one' Mendelian inconsistency has been recorded. Finally, we compared the contingency tables produced using Fisher's exact test.

SNP array genotypes, sample and variant pruning. Illumina GoatSNP50 BeadChip genotypes specifically generated for this research and Illumina OvineHD Beadchip genotyping data generated by two previous studies ${ }^{8,10}$ were considered in the analyses. Polled (i.e hornless) animals were removed from the sheep dataset. Markers with a minor allele frequency below $5 \%$ or which were called in less than $95 \%$ of the samples were eliminated. Moreover, in sheep, genotyping data were extracted for markers located in a $10 \mathrm{Mb}$ region $(\mathrm{Chr} 2: 127,500,001$ $138,500,000)$ corresponding approximately to the $H O X D$ gene cluster $+/-5 \mathrm{Mb}$ and encompassing all the mapping intervals of the POLYCERATE locus reported in the literature (Greyvenstein et al. 2016; He et al. 2016; Kijas et al. 2016; Ren et al. 2016). The final datasets contained 111 cases, 87 controls and 2’232 markers in sheep and 35 cases, 51 controls and 48’345 markers in goat.

\section{Analysis of Whole-Genome Sequences}

Whole-genome sequences. The genomes of one polycerate Provençale goat and one polycerate Jacob sheep were sequenced specifically for this study. Both were born from polycerate X wild type crosses and thus were predicted to be heterozygous for the caprine and ovine causative variants, respectively. Paired-end libraries with a 450 bp (goat) and 235 bp (sheep) insert size were generated using the NEXTflex PCR-Free DNA Sequencing Kit (Biooscientific). Libraries were quantified with the KAPA Library Quantification Kit (Cliniscience), controlled on a High Sensitivity DNA Chip (Agilent) and sequenced on a HiSeq 2500 (with $2 * 100$ bp read length in goat) and a HiSeq 3000 (with $2 * 150 \mathrm{bp}$ read length in sheep). The average sequence coverage was 16.7 and $11.1 \mathrm{x}$, for the polycerate goat and sheep individuals, respectively. Additional whole-genome sequences available in public databases were also considered in the analyses. These consisted of FASTQ files (for 10 additional case and 341 control sheep) and of VCF files (for 1160 goat and 838 sheep control individuals) generated by previous studies (see Supplementary Table 13). When necessary, the NCBI Genome Remapping Service (https://www.ncbi.nlm.nih.gov/genome/tools/remap) was used to convert positions in VCF files between older and most recent versions of genome assemblies.

Read alignment, variant calling and filtering for candidate variants. The sequence reads from FASTQ files were mapped on goat ARS1 (https://www.ncbi.nlm.nih.gov/assembly/GCF_0017 04415.1/) and sheep Oar_v4.0 (https://www.ncbi.nlm.nih.gov/assembly/GCF_000298735.2) 
genome assemblies using the BWA-MEM software $\mathrm{v}$ 0.7.17 with default parameters ( $\mathrm{Li}$ and Durbin 2009) and converted to bam format with v 1.8 of SAMtools (Li et al. 2009). Duplicate reads were marked using Picard tools V 2.18.2 MarkDuplicates option (http://broadinstitute.github.io/picard) and base quality recalibration and indel realignments were done with v 3.7 of GATK (McKenna et al. 2010). Reads located in the mapping intervals of the ovine and caprine POLYCERATE loci $+/-1 \mathrm{Mb}$ were extracted using SAMtools view option before processing to the calling of SNPs and small indels with GATK-HaplotypeCaller in ERC mode. The minimum read mapping quality and phred-scaled confidence threshold were set to 30 for each sample ('-stand_call_conf 30.0 -mmq 30 -ERC GVCF -variant_index_type LINEAR -variant_index_parameter 128000'). In goats we retained only heterozygous variants found in the heterozygous polycerate individual and absent from 1160 control animals, while in sheep we focused our attention on variants which were shared (either in heterozygous or homozygous state) in all the 11 polycerate sheep (1 Jacob and 10 Sishui Fur Sheep) and absent from the 1179 control animals. Finally, to ensure that we did not miss any candidate variants, we performed a detection of structural variants in the same regions using Pindel (Ye et al. 2009) and a visual examination of the whole genome sequences for 11 goats ( 1 case, 10 controls $)$ and 22 sheep (11 cases and 11 controls) using IGV (Thorvaldsdóttir et al. 2013). The count command in IGVtools was used to produce '.tdf' files and identify changes in read coverage in the intervals investigated (with parameters: zoom levels $=10$, window function $=$ mean, window size $=1000$, and extension factor $=500$ ).

\section{Definition of the Boundaries of the 503 kb Deletion-137 kb Insertion in Goat}

The boundaries of variant g.115,652,290_116,155,699delins137kb were reconstructed manually using split read and paired-end read information obtained from IGV. Sequences of reads affected by the mutation were extracted from the .bam file using linux command lines and aligned manually to reconstruct the nucleotide sequence at each fusion point. For verification, amplicons encompassing these fusion points were PCR amplified in a Mastercycler pro thermocycler (Eppendorf) using Go-Taq Flexi DNA Polymerase (Promega), according to the manufacturer's instructions and primers listed in Supplementary Table 15. Amplicons were purified and bidirectionally sequenced by Eurofins MWG (Hilden, Germany) using conventional Sanger sequencing.

\section{Genotyping of DNA Sequence Variants}


SNP and small Indels were genotyped using PCR and Sanger sequencing as described above. PCR primers were designed with Primer3 software (Rozen and Skaletsky 1999) and variants were detected using NovoSNP software (Weckx et al. 2005). Transgene insertions and large insertion-deletion were genotyped by PCR and electrophoresis on a $2 \%$ agarose gel. Ovine variant g.132,832,249_132,832,252del was genotyped with primers TTTGGGGCCACACTAGAATC and CCTAGAGGGGGCCTACGAG while caprine and murine variants were genotyped with the primers listed in Supplementary Table 7 and 14 respectively.

\section{Analysis of Nucleotide Sequence Conservation at the HOXD1 Exon 1-Intron-1 Junction}

Nucleotide sequences of the HOXD1 gene in 103 sarcopterygian and tetrapod species were obtained from the Ensembl (http://www.ensembl.org/index.html; release 98) and UCSC (http://genome.ucsc.edu/) genome browser databases. The localization of the nucleotide sequence (between MTX2 and HOXD3) was verified in each genome assembly to avoid possible confusion with paralogs. In addition, only one sequence was arbitrarily retained when genome assemblies for distinct individuals of the same species were available. Then sequences were put in the same orientation and trimmed to get 40 nucleotides before and 20 nucleotides after the splice donor site of HOXD1 exon 1. A multispecies alignment was generated with ClustalW software (Thompson et al. 1994), version 2.1 (https://www.genome.jp/tools-bin/clustalw) and a sequence logo was generated using WebLogo (Crooks 2004) (http://weblogo.berkeley.edu/). Information on species, sequence and genome assemblies are presented in Supplementary Table 5.

\section{Fluorescence In Situ Hybridization in Goat}

Skin biopsies were sampled from one heterozygous polycerate and one wild-type fetuses. Fibroblast cultures and metaphases were obtained according to (Ducos et al. 2000). Nucleotide sequences from the segments of caprine chromosomes 2 and 5 involved in the candidate causative mutation were aligned against bovine bacterial artificial chromosome (BAC) end sequences using BLAST (http://blast.ncbi.nlm.nih.gov/Blast.cgi). Two INRA BAC clones (Eggen et al. 2001) were selected and obtained from the Biological Resources of @BRIDGe facilities (abridge.inrae.fr): INRAb 230B11, targeting the segment deleted on Chr2, and INRAb 348A12, targeting the region of Chr5 that is duplicated and inserted on Chr2. FISH experiments were carried out according to (Yerle et al. 1994). The two BACs were labeled with biotin and digoxygenin, respectively, using the BioPrime DNA Labeling System kit (Life Technologies, 
Carlsbad, CA, USA). Finally, they were revealed by Alexa 594 conjugated to streptavidin (Molecular Probes, Eugene, OR, USA) and FITC conjugated mouse anti-digoxygenin antibodies (Sigma, St Louis, MO).

\section{Histological Analyses}

Tissues were fixed in paraformaldehyde $(4 \%)$ for $24 \mathrm{~h}$ at $+4^{\circ} \mathrm{C}$. Samples were subsequently dehydrated in a graded ethanol series, cleared with xylene and embedded in paraffin wax. Microtome sections ( $5 \mu \mathrm{m}$, Leica RM2245) were mounted on adhesive slides (Klinipath- KPPRINTER ADHESIVES), deparaffinized, and stained with haematoxylin, eosin and saffron (HES). Slides were scanned with the Pannoramic Scan 150 (3D Histech) and analyzed with the CaseCenter 2.9 viewer (3D Histech).

\section{Quantitative RT-PCR}

RNA was extracted using the RNeasy Mini Kit (Qiagen). Super-Script II (Invitrogen) was used to synthesize cDNA from $2 \mu \mathrm{g}$ of total RNA isolated from each tissue sampled in $70 \mathrm{dpc}$ goat and $76 \mathrm{dpc}$ sheep fetuses. Gene sequences were obtained from Ensembl v92 (www.ensembl.org) and PCR primers (Supplementary Table 16) were designed using Primer Express Software for Real-Time PCR 3.0 (Applied Biosystems). Primer efficiency and specificity were evaluated on genomic DNA in each species. Quantitative PCR was performed in triplicate with 2 ng of cDNA using the Absolute Blue SYBR Green ROX mix (Thermo Fisher Scientific) and the StepOnePlus Real-Time PCR System (Applied Biosystems). The expression stability of five genes (RPLP0, GAPDH, H2AFZ, YWHAZ and HPRT1) was tested at each time point using the GeNorm program (Vandesompele et al. 2002) to identify appropriate qRT-PCR normalizing genes. Three normalizing genes (GAPDH, H2AFZ and HPRT1) were retained and the results were analyzed with qBase software (Hellemans et al. 2007).

\section{Evaluation of the Consequences of Intron Retention Due to the 4 bp Deletion in HOXD1 intron 1}

The complete nucleotide sequence of ovine HOXD1 gene was obtained from Ensembl v97. A mutant mRNA characterized by (i) a retention of intron 1 and (ii) a deletion of nucleotides located at position +4 to $+7 \mathrm{bp}$ after the end of exon 1 was designed. This mutant mRNA was translated using ExPASy Translate tool (https://web.expasy.org/translate/). Information on HOXD1 functional domains was obtained from UniProt Knowledgebase (https://www.uniprot.org/uniprot/W5Q7P8). 


\section{D Geometric Morphometrics}

Three-dimensional models. Three-dimensional models were generated for 80 skulls consisting of 32 polycerate and 29 wild type sheep specimens as well as 12 polycerate and 7 wild type goat specimens (for information on skulls and reconstruction methods see Supplementary Table 10). Most of the 3D models $(n=47)$ were reconstructed using a Breuckmann StereoScan structured light scanner and its dedicated software OptoCat (AICON 3D systems, Meersburg, Germany). Twenty-nine skulls were digitized with the Artec Eva structured-light scanner and ScanStudioHD software v12.1.1.12 (Artec 3D, Luxembourg, Luxembourg). In addition, four skulls were digitized with a photogrammetric approach, similar to that described in (Evin et al. 2016). In brief, hundred pictures per sample were taken on different angles and inclinations with a Nikon D3300 camera equipped with an AF-S Micro Nikkor 85mm lens (Nikon, Tokyo, Japan) and a self-made fully automatic turntable. Then 3D models were reconstructed with the ReCap Photo software (Autodesk, San Rafael, CA, USA). Previous studies indicated no significant differences between 3D models obtained with three-dimensional scanners or photogrammetry (Evin et al. 2016; Fau et al. 2016). Both approaches are comparable in terms of measurement error (less than $1 \mathrm{~mm}$ ). Bone surfaces were extracted as meshes and geometric inconsistencies (i.e. noise, holes) were cleaned using Geomagic software (3D Systems, Rock Hill, USA).

Shape analyses. 116 3D landmarks and sliding semi-landmarks were placed on each specimen by the same operator using the IDAV Landmark software (Wiley et al. 2005) v3.0. Out of them 16 were anatomical landmarks, and 100 were sliding semi-landmarks individually placed around the basis of the horns on the suture between the bony core and the frontal bone. On each side, the first of these 50 sliding semi-landmarks was placed on the upper horn, at the intersection between the upper ridge of the bony core and the suture previously mentioned. Details on landmark locations on polycerate and wild type specimens are provided in Supplementary Table 11 and Supplementary Fig. 11.

Following the procedure detailed by (Botton-Divet et al. 2015), a template was created using the specimen 2000-438 on which all anatomical landmarks and surface sliding semi-landmarks were placed. Then, a semi-automatic point placement was performed (Gunz and Mitteroecker 2013) to project sliding semi-landmarks on the surface of the other 3D digitized skulls. Sliding semi-landmarks on surfaces and curves were allowed to slide in order to minimize the bending energy of a thin plate spline (TPS) between each 3D meshes and the template. After this first 
525 TPS relaxation using the template, three iterative relaxations were performed using the Procrustes consensus of the previous step as a reference.

527 To remove non-shape variation (i.e. differences in position, scale, and orientation of the

528 configurations) and provide optimal comparability between the specimens, we performed a generalized Procrustes Analysis (GPA) (Rohlf and Slice 1990). Since our dataset contained more variables than observations, we performed a Principal Component Analysis (PCA) on the procrustes residuals to reduce dimensionality, as recommended by (Gunz and Mitteroecker 2013), and plotted the first Principal Components (PCs) to visualize the specimen distribution in the morphospace. In addition, the mean shape of our sample was used to compute theoretical shapes associated with the maximum and minimum of both sides of the first PC axis for each species using thin plate spline. GPA, PCA and shape computations were done using the 'Morpho' and 'geomorph' packages (Adams and Otárola-Castillo 2013; Adams et al. 2018; Schlager 2018) in the R environment (R Core Team 2018).

Repeatability and reproducibility of landmark placement. The 116 landmarks and sliding semilandmarks were placed ten times independently on the skulls from two polycerate and two control male sheep sampled between 1852 and 1909 in Tunisia (A-12130, A12132, 1909-4) and neighboring Algeria (A12157; see Supplementary Table 10). The measurements were superimposed using a GPA and analyzed using a PCA. Since the variation within specimens was clearly smaller than the variation between specimens (Supplementary Fig. 12), we considered that the 116 landmarks and sliding semi-landmarks were precise enough to describe shape variation.

\section{Data Availability}

547 Raw sequencing data that support the findings of this study have been deposited to the European Variation Archive (EVA, https://www.ebi.ac.uk/eva/) under accession number PRJEB39341. Sequences from previous studies can be found at the following URL (www.goatgenome.org/vargoats_data_access.htm) or in the NCBI BioProject and EVA databases under accession numbers PRJEB6025, PRJEB6495, PRJEB9911, PRJEB14098, PRJEB14418，PRJEB15642， PRJEB23437， PRJEB31241，PRJEB31930， PRJEB32110, PRJEB35553, PRJEB35682, PRJEB37460, PRJEB39341, PRJEB39341 and PRJNA624020. Illumina GoatSNP50 Beadchip genotyping data generated for this study have been deposited in the Dryad Digital Repository (doi: 10.5061/dryad.rxwdbrv6n). Illumina OvineHD Beadchip genotyping data from previous studies can be found in the same repository (doi: 
10.5061/dryad.6t34b and 10.5061/dryad.1p7sf). Coordinates of landmarks and source data underlying Fig. 3 and 4, and Suppl. Fig. 2, 10 and 11 are provided as a Source Data file.

\section{Acknowledgements}

We thank L. Orlando (Universities of Toulouse III, France and Copenhagen, Denmark) for tentatively extracting DNA from museum skull specimens, as well as C. Hozé, F. Lejuste and A Michenet (ALLICE), R. McCulloch (CSIRO), S. Chahory and C. Degueurce (ENVA), F. Andreoletti, M. Boussaha, J. Kergosien, D. Mauchand, M. Femenia, N. Perrot and M. Vilotte (INRAE), B. Camus-Allanic (LABOGENA DNA), J. Peters (LMU), C. Bens, A. Delapré and A.Verguin (MNHN), L. Ludes-Fraulob (MZS) and B. Mascrez (University of Geneva) for their assistance. We also thank the staff of the INRAE experimental unit UE 1298 SAAJ for animal husbandry and management, as well as breeders and zoological parcs for making animals available for sampling and for providing pictures. Contributors include in particular the Capgènes breeding company (France), L. Pachot (Mouton Village, Vasles, France), A. Archiloque (France), L. Fiorenzi (Az. Agr. Madonna delle Alpi, Italy), the Schafzuchtverein Jakobschaf Schweiz (Switzerland), Tierpark Hamm (Germany), A. Schumann (Germany) and Dr. A. Ennaifer (Zoological Park of Tunis, Tunisia). Finally, the authors thank the VarGoats Consortium for allowing variant filtering against their dataset. The Vargoats project was supported by France Génomique (grant number ANR-10-INBS-0009). This work was supported by APIS-GENE (grant AKELOS) and the Swiss National Research Foundation (grant number No 310030B_138662 to D.D.). A.Hi. was supported by a PhD fellowship from the University of Geneva.

\section{Authors contributions}

O.G., D.R., T.H., N.C., C.M.R., B.J.H and J.K. provided Illumina OvineHD Beadchip genotyping data and related phenotypes. A.C. mapped the ovine and caprine polycerate loci. C.Dr., C.D.-B., D.B., I.M, L.P., O.G., T.H., G.B., F.M., N.H., J.P., S.B.J., J.H., R.R., I.P., J.A.L., L.G., D.R., E.V.M.-K., N.C., B.J.H, J.K. and G.T.-K. provided samples and phenotypes. D.E. and C.Do. performed whole genome sequencing from one polycerate Provençale goat and one polycerate Jacob sheep. G.T.-K. provided control whole genome sequences from sheep and goats. A.C., P.B., and M.N.-S. performed variant calling, annotation and screening for candidate variants. A.C. and A.A.-B. analysed sequence conservation and annotated the gene content of the mapping intervals. M.-C.D., C.Gr. and A.Hi. extracted DNA. M.-C.D., A.C., C.Gr., and A.Hi. performed PCR for Sanger sequencing and for genotyping by PCR and 
electrophoresis or PCR and Sanger sequencing. A.P. performed FISH analysis. A.Hi., J.Z and D.D. produced and studied mouse models. E.P. provided access to laboratory and experimental farm facilities. A.C., A.A.-B., M.-C.D., C.Gr. and E.P. sampled ovine and caprine fetuses. A.A.B. and M.-C.D. extracted RNA, performed qRT-PCR and analysed the results. A.Bo., J.R., A.C., A.A.-B. and M.-C.D. performed histological analyses. A.C., M.S. and A.Ha. performed 3D data acquisition of skulls. A.B1. provided access to a light scanner for 3D data acquisition. O.P., J.L., R.S., M.-D.W., R.-M.A. and C.Gu. provided access to skull specimens and related information. A.C. performed morphometric analyses. R.C. provided software and expertise in morphometric analyses. A.C. (Bovidae) and D.D. (mouse) designed the studies and wrote the manuscript, which was accepted or revised by all authors.

\section{Figure Legends}

Figure 1. Polyceraty in sheep and goats and candidate genetic variants. a) Polycerate Manx Loaghtan ram. b) Wildtype and polycerate male goats from a local German population. These individuals represent the most common phenotype. Polycerate animals with asymetric horns and partial fusion of lateral horns are also regularly observed. c) A 4bp deletion causing polyceraty in sheep. Integrative Genome Viewer (IGV) screenshot with the localization of the variant with respect to HOXD1. Below is a graphical representation of nucleotide conservation at the exon 1-intron junction across 103 sarcopterigian and tetrapod species. d) Plot of read coverage in a heterozygous polycerate goat animal carrying a deletion of $503 \mathrm{~kb}$ downstream the HOXD gene cluster on Chr2 and a duplication of $137 \mathrm{~kb}$ on Chr5. e) FISH-mapping in a heterozygous polycerate goat with $\mathrm{BAC}$ clones corresponding to the region deleted in $\mathrm{Chr} 2$ (labeled in red) and to the segment of Chr5 inserted at the deletion site (labeled in green). Magnification: X1000. Sheep and goat icons were made by 'Monkik' from www.thenounproject.com.

Figure 2. Regulation of Hoxd1 expression pattern in crest cell-derived head structures in mouse. a) On top is the structure of the mouse $\operatorname{HoxD}$ gene cluster with arrows showing the timing and localisation of gene expression along the body axis during development. The position of Hoxdl is highlighted in red. Below is a $1 \mathrm{Mb}$ view of the locus, with Hoxdl in red as well as the relative position of the POLYCERATE variants in sheep (black arrowhead) and goat (black line). Below are depicted the various murine alleles, with the lacZ insertion in Hoxdl (blue arrowhead), the two BAC clones (thick blue lines) and the engineered deletion (black line). b) Fetal heads of E12.5-E13.5 mouse fetuses after X-gal staining. The dashed circle highlights the absence of Hoxdl expression in the crown (corresponding to the localization of 
hornbuds in Bovidae), whereas the surrounding dermal cells are positive. The conservation of Hoxd 1 expression in the back of the neck (black arrows) contrasts with the presence/absence of expression in the facial muscle precursors (white arrows) and in the eyelids (arrowhead). The comparison between the four strains indicate that Hoxdl expression in all these cranial derivatives is controlled by regulatory elements located in a region orthologous to the proximal portion of the segment deleted in polycerate goats.

Figure 3. RT-qPCR gene expression analyses in sheep and goat foetuses. a, b) Schemes of the tissues sampled at stage $70 \mathrm{dpc}$ in goat (a) and $76 \mathrm{dpc}$ in sheep (b) in four control (+/+) and four heterozygous (+/-) polycerate fetuses within each species. bs: skin from the back of the head; hb: skin from the hornbud; h1: skin from the lower horn bud; and h2: skin from the upper horn bud in polycerate specimens; fs: frontal skin; el: eyelids. RT-qPCR gene expression analyses in these tissues are shown below (means and standard errors of the means). *: p<0.05, **: $\mathrm{p}<0.01$ (Welch two sample t-test with the alternative hypothesis that the means are not equal). For the sake of clarity, the symbols \# and @ were also used to show significant differences $(\mathrm{p}<0.05)$ between distant bars.

Figure 4. Results of three-dimensional geometric morphometric analyses of 61 ovine and 19 caprine skulls. a) Distribution of the specimens along the first two axes of the PCA. The proportion of variance explained by the main principal components is indicated on each axis. Green dots: polycerate sheep with a distance between lateral horns (dlh) larger than the distance between upper horns (duh); light blue: polycerate sheep with a dlh $\leq \mathrm{duh}$; blue: polycerate sheep with at least two lateral horns partially fused at their basis; purple: wild type sheep; black: polycerate goats; and red: wild type goats. Representative specimens illustrate each cluster and symbols are used to indicate their respective locations in the PCA analysis (see Suppl. Fig. 10 for intraspecies analyses and further information). b) Number of heterozygous (+/-) and homozygous (-/-) polycerate rams amongst groups of live animals with different dlhl (dlh on the left side) and duh relative sizes (see Suppl. Table 12 for further information); ${ }^{* * *}$ : p-value= $3.5 \times 10^{-7}$ (Fisher's exact test). c) Theoretical shapes associated with the maximum (upper three) and minimum values (lower three) of PC1 axis for a sheep skull. Red dots correspond to anatomical landmarks while the other dots correspond to sliding semi-landmarks; light blue and purple dots highlight the sites of division of lateral horns. d) Shape differences for the sliding semi-landmarks located at the basis of the left horn. Light blue and purple dots correspond to the maximum and minimum values of PC1 axis, respectively. Dashed squares indicate the 
estimated position of dissected tissues in Fig. 3 (bs: skin from the back of the head; fs: frontal skin) in which HOXD1 expression was observed in fetuses.

\section{References}

Adams DC, Collyer M, Kaliontzopoulou A. 2018. Geometric Morphometric Analyses of 2D/3D Landmark Data. Available from: http://kambing.ui.ac.id/cran/web/packages/geomorph/geomorph.pdf.

Adams DC, Otárola-Castillo E. 2013. geomorph: an r package for the collection and analysis of geometric morphometric shape data.Paradis E, editor. Methods Ecol Evol 4:393-399.

Allais-Bonnet A, Grohs C, Medugorac I, Krebs S, Djari A, Graf A, Fritz S, Seichter D, Baur A, Russ I, et al. 2013. Novel Insights into the Bovine Polled Phenotype and Horn Ontogenesis in Bovidae. Yue J, editor. PLoS ONE 8:e63512.

Andrey G, Montavon T, Mascrez B, Gonzalez F, Noordermeer D, Leleu M, Trono D, Spitz F, Duboule D. 2013. A switch between topological domains underlies HoxD genes collinearity in mouse limbs. Science 340:1234167.

Bickhart DM, Rosen BD, Koren S, Sayre BL, Hastie AR, Chan S, Lee J, Lam ET, Liachko I, Sullivan ST, et al. 2017. Single-molecule sequencing and chromatin conformation capture enable de novo reference assembly of the domestic goat genome. Nature Genetics 49:643-650.

Botton-Divet L, Houssaye A, Herrel A, Fabre A-C, Cornette R. 2015. Tools for quantitative form description; an evaluation of different software packages for semi-landmark analysis. PeerJ 3:e1417.

Capitan A, Allais-Bonnet A, Pinton A, Marquant-Le Guienne B, Le Bourhis D, Grohs C, Bouet S, Clément L, Salas-Cortes L, Venot E, et al. 2012. A 3.7 Mb Deletion Encompassing ZEB2 Causes a Novel Polled and Multisystemic Syndrome in the Progeny of a Somatic Mosaic Bull.Yue J, editor. PLoS ONE 7:e49084.

Capitan A, Grohs C, Weiss B, Rossignol M-N, Reversé P, Eggen A. 2011. A Newly Described Bovine Type 2 Scurs Syndrome Segregates with a Frame-Shift Mutation in TWIST1.Toland AE, editor. PLoS ONE 6:e22242.

Crooks GE. 2004. WebLogo: A Sequence Logo Generator. Genome Research 14:1188-1190.

Davis EB, Brakora KA, Lee AH. 2011. Evolution of ruminant headgear: a review. Proc. R. Soc. B. 278:2857-2865.

Dove WF. 1935. The physiology of horn growth: a study of the morphogenesis, the interaction of tissues and the evolutionary processes of a Mendelian recessive character by means of transplantation of tissues. Journal of Experimental Zoology:347-405.

Ducos A, Dumont P, Séguéla A, Pinton A, Berland H, Brun-Baronnat C, Darré A, MarquantLe Guienne B, Humblot P, Boichard D, et al. 2000. A new reciprocal translocation in a subfertile bull. Genetics, selection, evolution: GSE 32:589-598. 
Durkin K, Coppieters W, Drögemüller C, Ahariz N, Cambisano N, Druet T, Fasquelle C, Haile A, Horin P, Huang L, et al. 2012. Serial translocation by means of circular intermediates underlies colour sidedness in cattle. Nature 482:81-84.

Eggen A, Gautier M, Billaut A, Petit E, Hayes H, Laurent P, Urban C, Pfister-Genskow M, Eilertsen K, Bishop MD. 2001. Construction and characterization of a bovine BAC library with four genome-equivalent coverage. Genetics, selection, evolution: GSE $33: 543-548$.

Epstein H. 1971. The origin of the domesticated animals of Africa. Africana Publishing Corporation. New York

Evin A, Souter T, Hulme-Beaman A, Ameen C, Allen R, Viacava P, Larson G, Cucchi T, Dobney K. 2016. The use of close-range photogrammetry in zooarchaeology: Creating accurate 3D models of wolf crania to study dog domestication. Journal of Archaeological Science: Reports 9:87-93.

Fau M, Cornette R, Houssaye A. 2016. Photogrammetry for 3D digitizing bones of mounted skeletons: Potential and limits. Comptes Rendus Palevol 15:968-977.

Frohman MA, Martin GR. 1992. Isolation and analysis of embryonic expression of Hox-4.9, a member of the murine labial-like gene family. Mechanisms of Development 38:55-67.

Gregory SG, Sekhon M, Schein J, Zhao S, Osoegawa K, Scott CE, Evans RS, Burridge PW, Cox TV, Fox CA, et al. 2002. A physical map of the mouse genome. Nature 418:743750 .

Greyvenstein OFC, Reich CM, van Marle-Koster E, Riley DG, Hayes BJ. 2016. Polyceraty (multi-horns) in Damara sheep maps to ovine chromosome 2. Anim Genet 47:263-266.

Groves C. 2003. Taxonomy of ungulates of the Indian subcontinent. Journal of the Bombay Natural History Society:314-362.

Gunz P, Mitteroecker P. 2013. SEMILANDMARKS: A METHOD FOR QUANTIFYING CURVES AND SURFACES. Hystrix, the Italian Journal of Mammalogy [Internet] 24. Available from: https://doi.org/10.4404/hystrix-24.1-6292

He X, Zhou Z, Pu Y, Chen X, Ma Y, Jiang L. 2016. Mapping the four-horned locus and testing the polled locus in three Chinese sheep breeds. Anim Genet 47:623-627.

Heitzmann A. 2006. Trianon, la ferme du Hameau. Versalia:114-129.

Hellemans J, Mortier G, De Paepe A, Speleman F, Vandesompele J. 2007. qBase relative quantification framework and software for management and automated analysis of realtime quantitative PCR data[No title found]. Genome Biol 8:R19.

Kijas JW, Hadfield T, Naval Sanchez M, Cockett N. 2016. Genome-wide association reveals the locus responsible for four-horned ruminant. Anim Genet 47:258-262.

Kmita M, Duboule D. 2003. Organizing axes in time and space; 25 years of colinear tinkering. Science 301:331-333.

Krumlauf R. 1994. Hox genes in vertebrate development. Cell 78:191-201. 
Li H, Durbin R. 2009. Fast and accurate short read alignment with Burrows-Wheeler transform. Bioinformatics (Oxford, England) 25:1754-1760.

Li H, Handsaker B, Wysoker A, Fennell T, Ruan J, Homer N, Marth G, Abecasis G, Durbin R, Genome Project Data Processing S. 2009. The Sequence Alignment/Map format and SAMtools. Bioinformatics 25:2078-2079.

Lincoln GA. 1973. Appearance of antler pedicles in early foetal life in red deer. Journal of Embryology and Experimental Morphology 29:431-437.

McKenna A, Hanna M, Banks E, Sivachenko A, Cibulskis K, Kernytsky A, Garimella K, Altshuler D, Gabriel S, Daly M, et al. 2010. The Genome Analysis Toolkit: a MapReduce framework for analyzing next-generation DNA sequencing data. Genome Research 20:1297-1303.

Putelat O. 2005. Le bestiaire polycère. Revue de Paléobiologie:293-301.

R Core Team. 2018. R: a language and environment for statistical computing. $\mathrm{R}$ foundation for statistical computing, Vienne. Available from: https://www.R-project.org/

Ren X, Yang G-L, Peng W-F, Zhao Y-X, Zhang M, Chen Z-H, Wu F-A, Kantanen J, Shen M, Li M-H. 2016. A genome-wide association study identifies a genomic region for the polycerate phenotype in sheep (Ovis aries). Sci Rep 6:21111.

Rohlf FJ, Slice D. 1990. Extensions of the Procrustes Method for the Optimal Superimposition of Landmarks. Systematic Zoology 39:40.

Rozen S, Skaletsky H. 1999. Primer3 on the WWW for General Users and for Biologist Programmers. In: Bioinformatics Methods and Protocols. Vol. 132. New Jersey: Humana Press. p. 365-386. Available from: http://link.springer.com/10.1385/1-59259192-2:365

Schep R, Necsulea A, Rodriguez-Carballo E, Guerreiro I, Andrey G, Nguyen Huynh TH, Marcet V, Zakany J, Duboule D, Beccari L. 2016. Control of Hoxd gene transcription in the mammary bud by hijacking a preexisting regulatory landscape. Proceedings of the National Academy of Sciences of the United States of America 113:E7720-E7729.

Schlager S. 2018. Morpho: calculations and vizualisations related to Geometric Morphometrics. Available from: https://cran.r-project.org/web/packages/Morpho/Morpho.pdf.

Thompson JD, Higgins DG, Gibson TJ. 1994. CLUSTAL W: improving the sensitivity of progressive multiple sequence alignment through sequence weighting, position-specific gap penalties and weight matrix choice. Nucleic Acids Research 22:4673-4680.

Thorvaldsdóttir H, Robinson JT, Mesirov JP. 2013. Integrative Genomics Viewer (IGV): highperformance genomics data visualization and exploration. Briefings in Bioinformatics $14: 178-192$.

Tosser-Klopp G, Bardou P, Bouchez O, Cabau C, Crooijmans R, Dong Y, Donnadieu-Tonon C, Eggen A, Heuven HCM, Jamli S, et al. 2014. Design and Characterization of a 52K SNP Chip for Goats.Liu Z, editor. PLoS ONE 9:e86227. 
bioRxiv preprint doi: https://doi.org/10.1101/2020.11.04.363069; this version posted November 4,2020 . The copyright holder for this preprint (which was not certified by peer review) is the author/funder, who has granted bioRxiv a license to display the preprint in perpetuity. It is made available under aCC-BY-NC-ND 4.0 International license.

Vandesompele J, De Preter K, Pattyn F, Poppe B, Van Roy N, De Paepe A, Speleman F. 2002. Accurate normalization of real-time quantitative RT-PCR data by geometric averaging of multiple internal control genes. Genome Biology 3:RESEARCH0034.

Wang Y, Zhang C, Wang N, Li Z, Heller R, Liu R, Zhao Y, Han J, Pan X, Zheng Z, et al. 2019. Genetic basis of ruminant headgear and rapid antler regeneration. Science 364:eaav6335.

Weckx S, Del-Favero J, Rademakers R, Claes L, Cruts M, De Jonghe P, Van Broeckhoven C, De Rijk P. 2005. novoSNP, a novel computational tool for sequence variation discovery. Genome Research 15:436-442.

Wiley DF, Amenta N, Alcantara DA, Ghosh D, Kil YJ, Delson E, Harcourt-Smith W, Rohlf FJ, St. John K, Hamann B. 2005. Evolutionary Morphing. In: VIS 05. IEEE Visualization, 2005. Minneapolis, MN, USA: IEEE. p. 431-438. Available from: http://ieeexplore.ieee.org/document/1532826/

Wilkins SJ, Yoong S, Verkade H, Mizoguchi T, Plowman SJ, Hancock JF, Kikuchi Y, Heath JK, Perkins AC. 2008. Mtx2 directs zebrafish morphogenetic movements during epiboly by regulating microfilament formation. Developmental Biology 314:12-22.

Ye K, Schulz MH, Long Q, Apweiler R, Ning Z. 2009. Pindel: a pattern growth approach to detect break points of large deletions and medium sized insertions from paired-end short reads. Bioinformatics 25:2865-2871.

Yerle M, Goureau A, Gellin J, Le Tissier P, Moran C. 1994. Rapid mapping of cosmid clones on pig chromosomes by fluorescence in situ hybridization. Mammalian Genome: Official Journal of the International Mammalian Genome Society 5:34-37.

Zakany J, Kmita M, Alarcon P, de la Pompa JL, Duboule D. 2001. Localized and transient transcription of Hox genes suggests a link between patterning and the segmentation clock. Cell 106:207-217. 
bioRxiv preprint doi: https://doi.org/10.1101/2020.11.04.363069; this version posted November 4, 2020. The copyright holder for this preprint (which was not certified by peer review) is the author/funder, who has granted bioRxiv a license to display the preprint in perpetuity. It is made available under aCC-BY-NC-ND 4.0 International license.

\section{Allais-Bonnet et al., Figure 1}

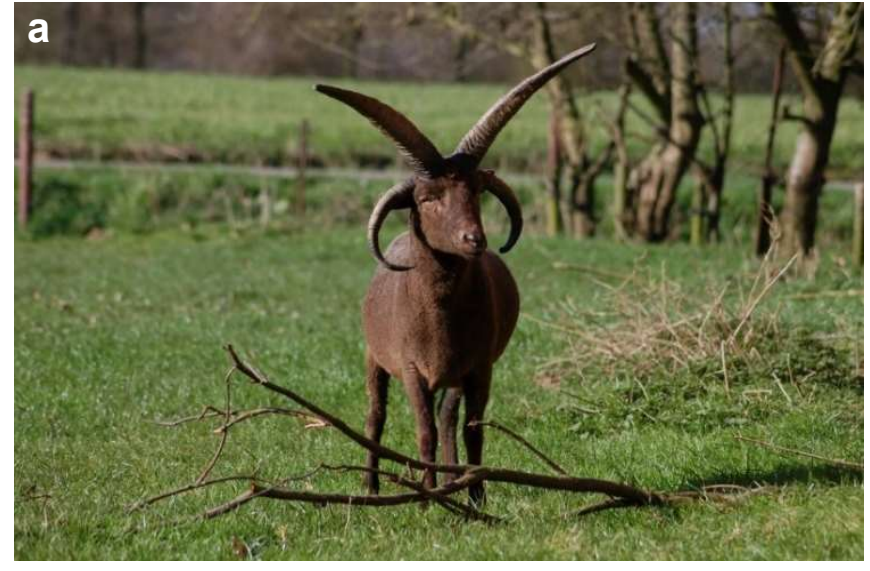

C

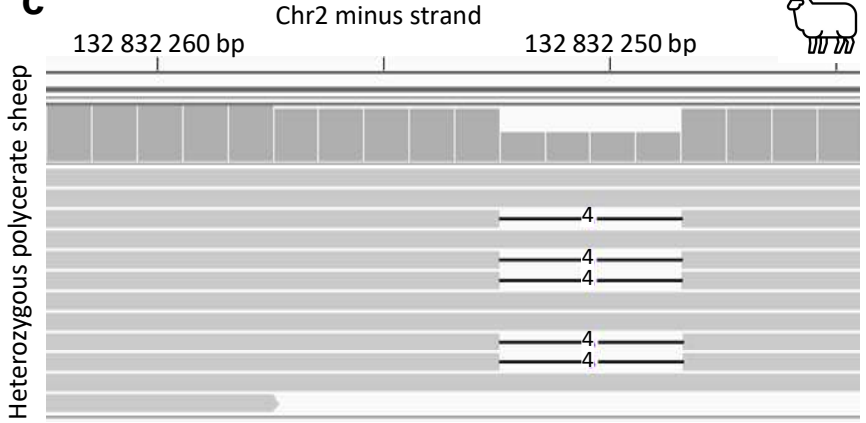

A A G A A A A G T A A $G$ T A $A$ C T
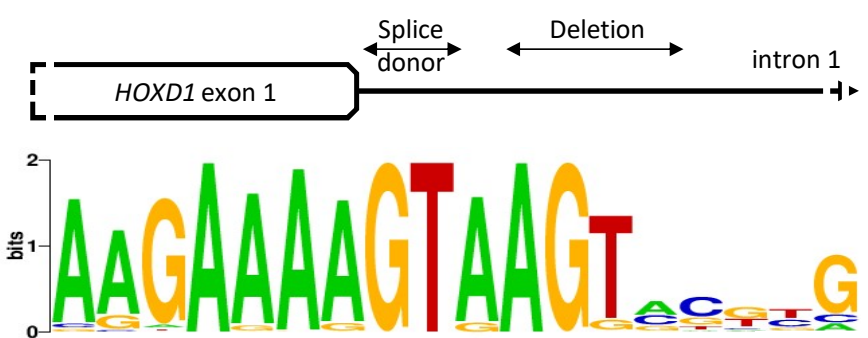

$+1+2+3+4+5+6+7$

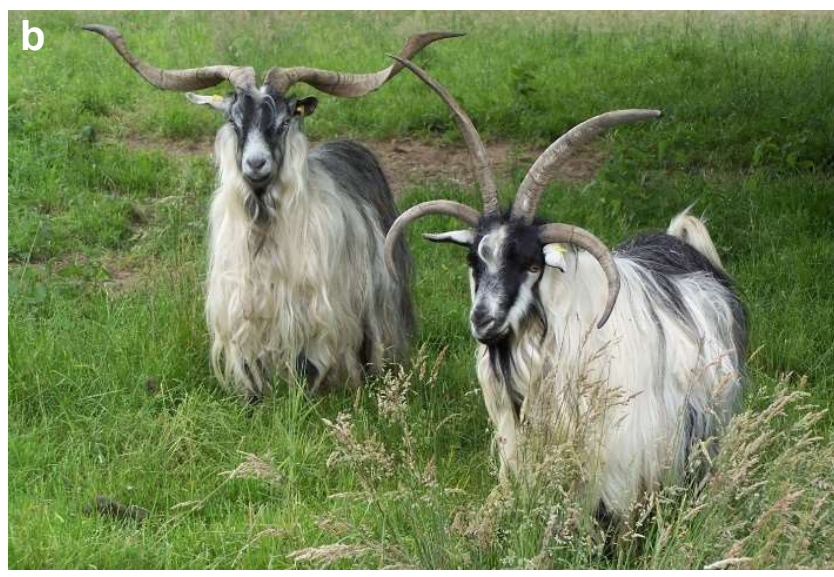

d
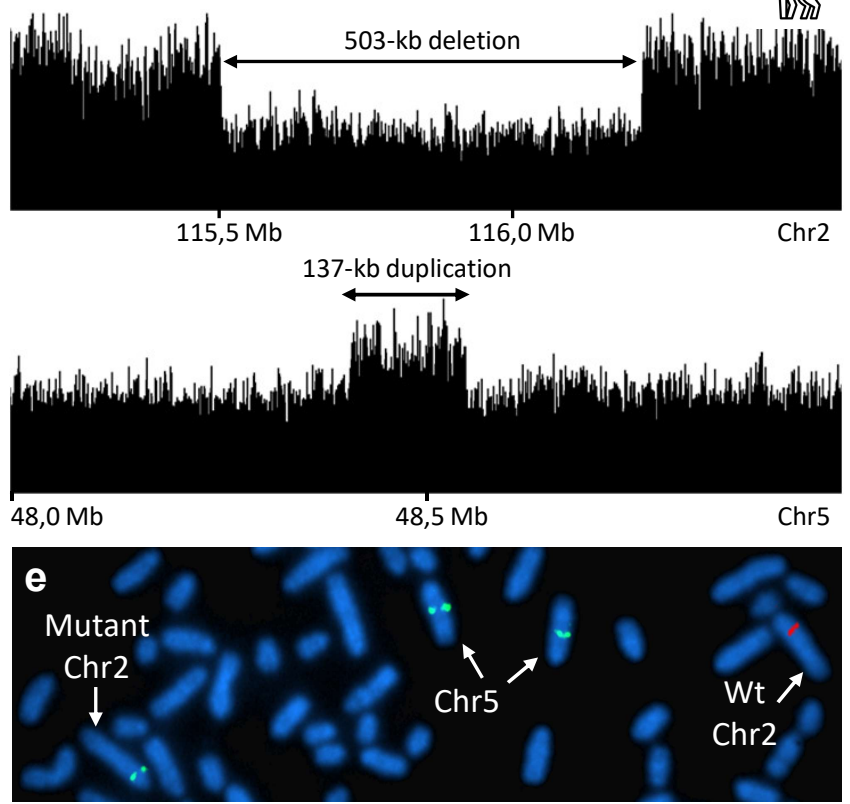
bioRxiv preprint doi: https://doi.org/10.1101/2020.11.04.363069; this version posted November 4, 2020. The copyright holder for this preprint (which was not certified by peer review) is the author/funder, who has granted bioRxiv a license to display the preprint in perpetuity. It is made available under aCC-BY-NC-ND 4.0 International license.

\section{Allais-Bonnet et al., Figure 2}

a
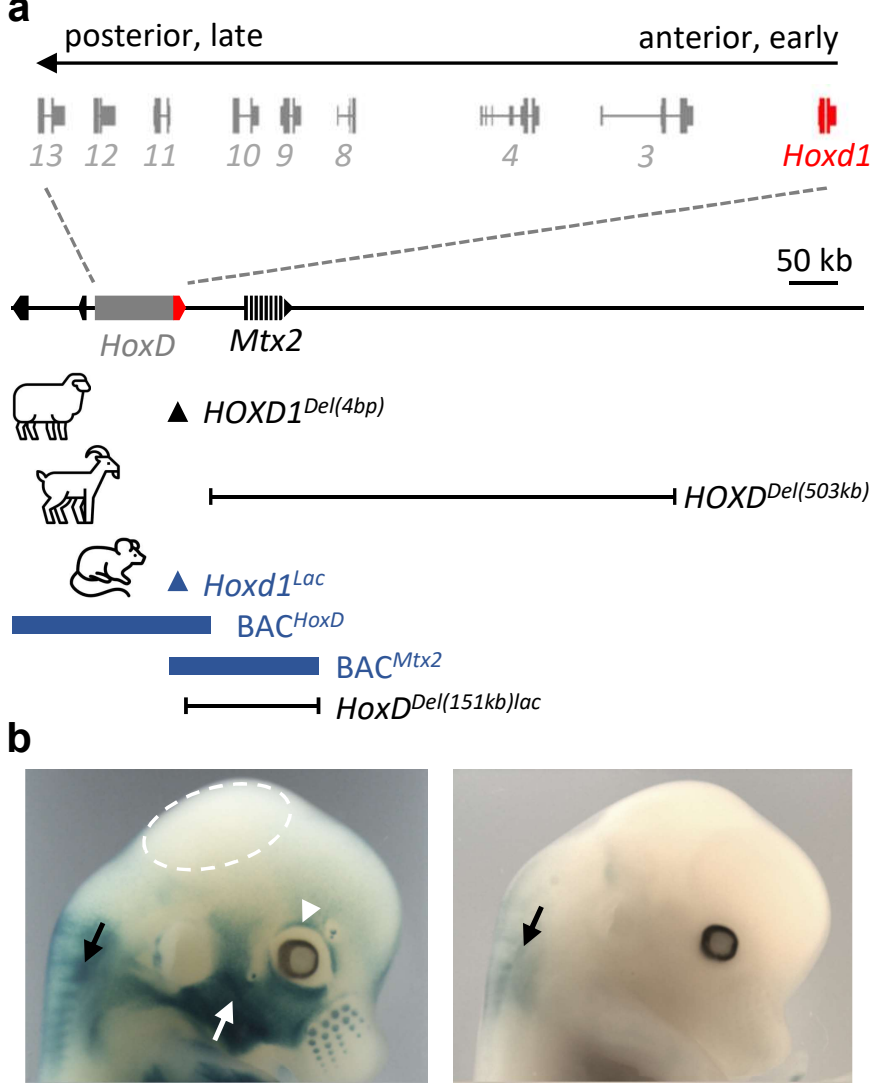

Hoxd1 ${ }^{\text {Lac }}$

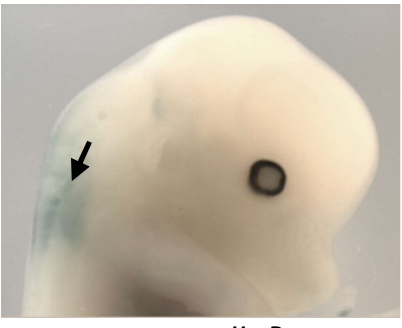

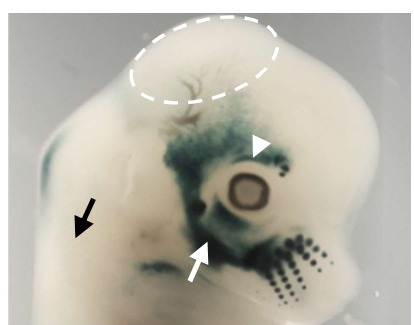

$T g B A C^{M t x 2}$
$\operatorname{TgBAC}{ }^{H o x D}$

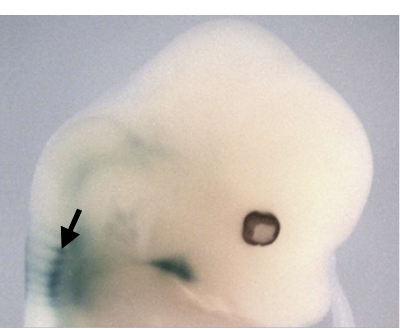

HoxD Del(151kb)lac 


\section{Allais-Bonnet et al., Figure 3}

a
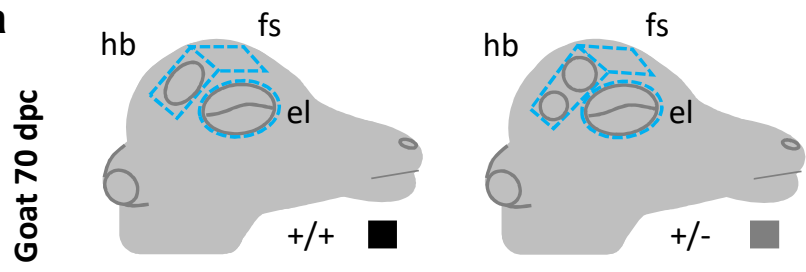

b
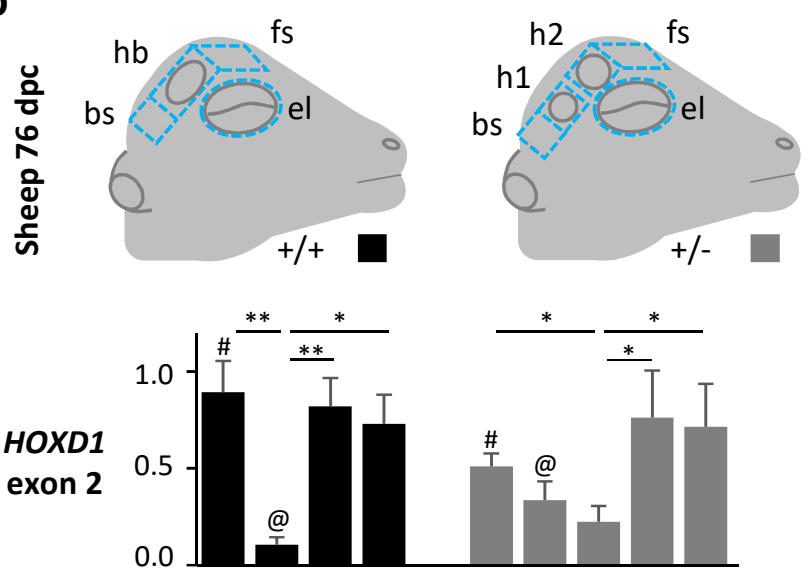

bs hb fs el bs h1 h2 fs el

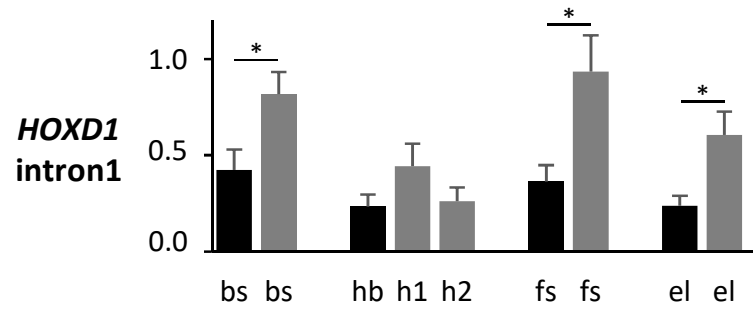


bioRxiv preprint doi: https://doi.org/10.1101/2020.11.04.363069; this version posted November 4, 2020. The copyright holder for this preprint (which was not certified by peer review) is the author/funder, who has granted bioRxiv a license to display the preprint in perpetuity. It is made available under aCC-BY-NC-ND 4.0 International license.

\section{Allais-Bonnet et al., Figure 4}

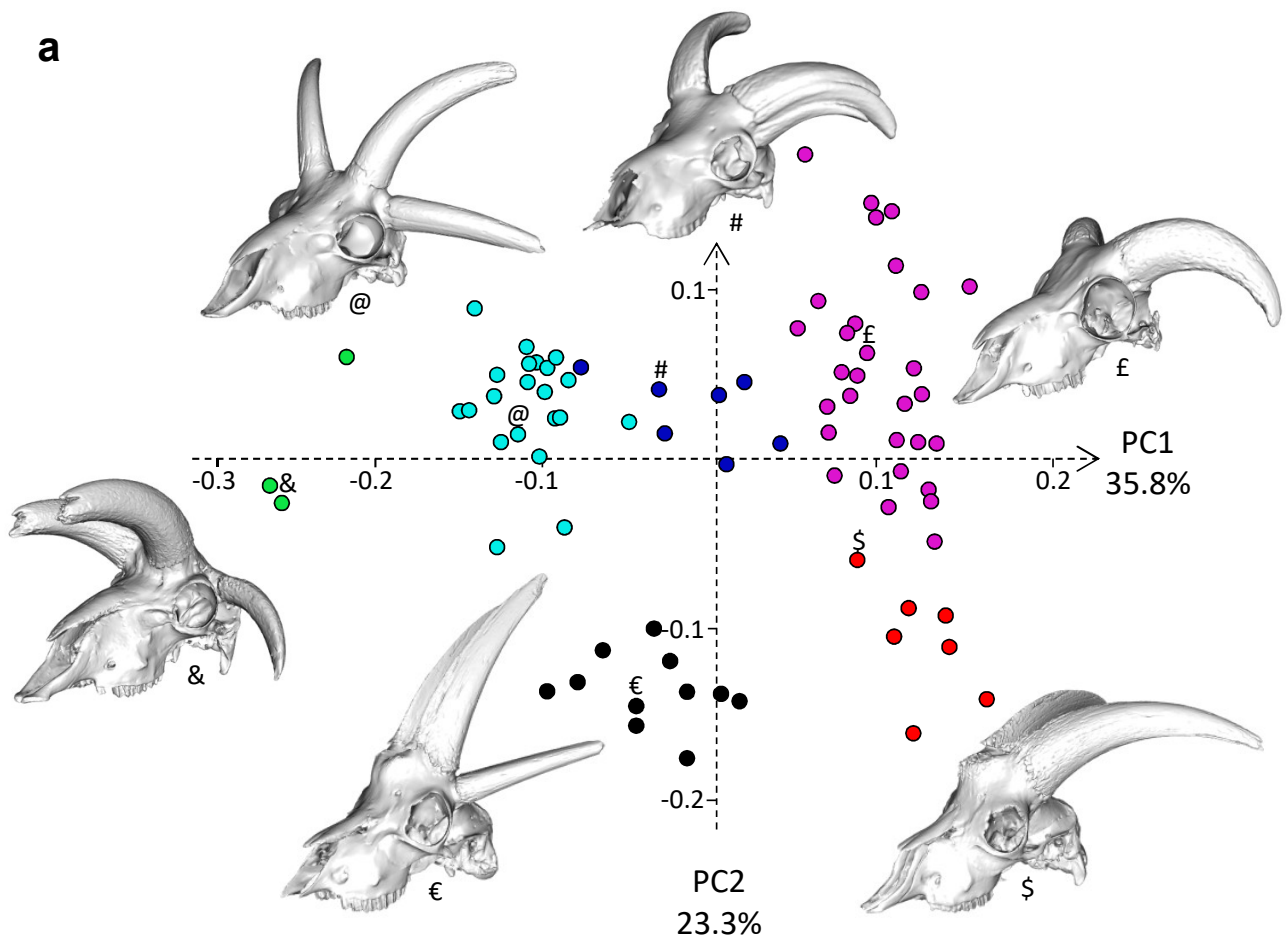

b Genotypes

$+/-\quad-\quad-$
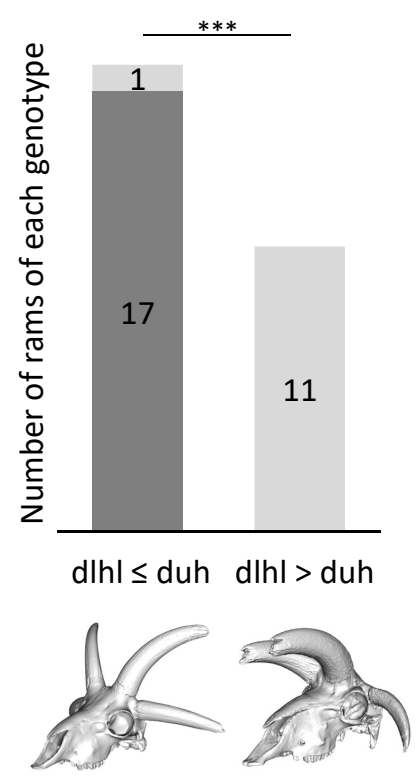
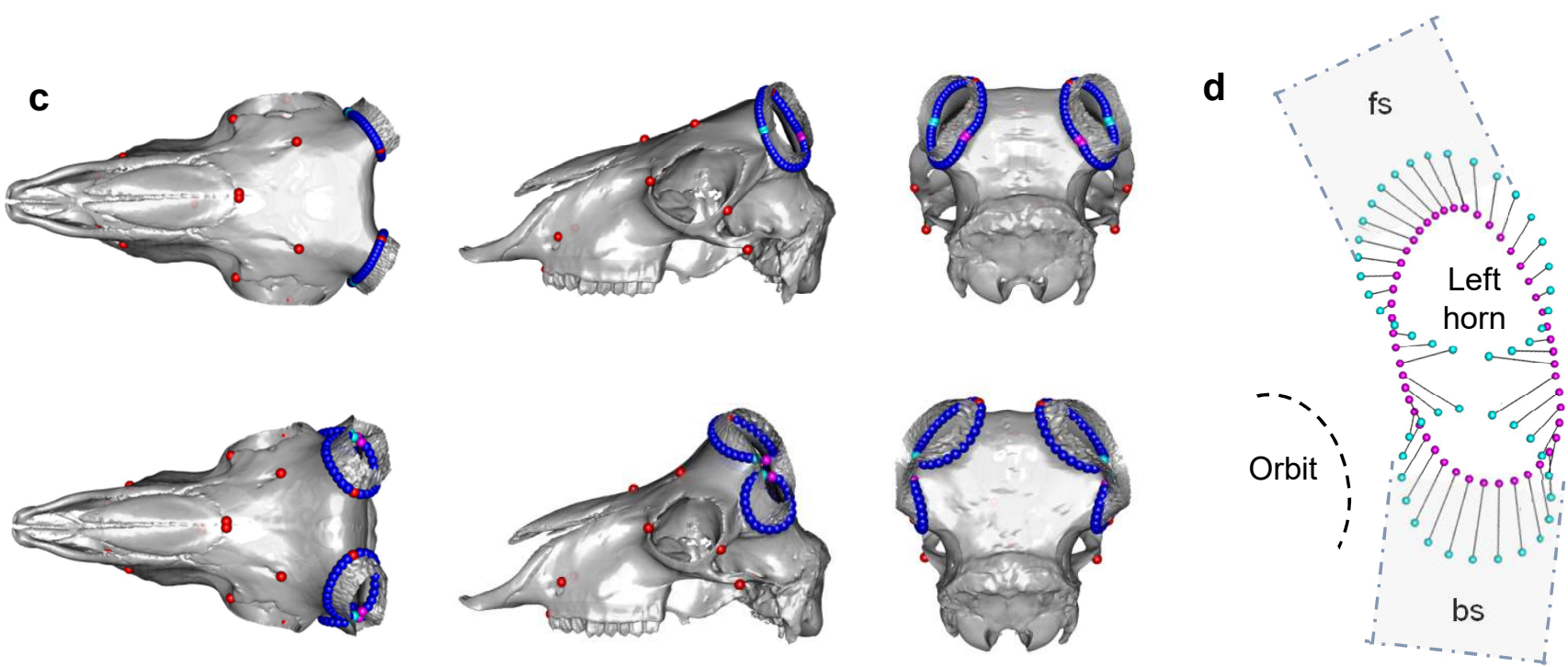